\title{
RELATED PROBLEMS IN PARTIAL DIFFERENTIAL EQUATIONS
}

BY L. R. BRAGG AND J. W. DETTMAN

Communicated by J. B. Diaz, October 18, 1967

1. Introduction. Let $x=\left(x_{1}, \cdots, x_{n}\right)$ and $D=\left(D_{1}, \cdots, D_{n}\right)$ where $D_{i} \phi(x)=\partial \phi(x) / \partial x_{i}$. Let $D^{\alpha}=D_{1}^{\alpha_{1}} D_{2}^{\alpha_{2}} \cdots D_{n}^{\alpha_{n}}$ and let $P(x, D)$ $=\sum_{\alpha ; 0 \leq|\alpha| \leq m} a_{\alpha}(x) D^{\alpha}$ where $|\alpha|=\alpha_{1}+\cdots+\alpha_{n}$ and the $a_{\alpha}(x)$ are given functions of $x$. Finally, let $S(x)=0$ denote a cylindrical surface in $(x, t)$ space and $B(x, D)$ a nontangential boundary operator whose domain is the manifold $S(x)=0$. The smoothness required of $S(x)=0$ will depend upon the operator $B(x, D)$. We will be concerned with the following pair of initial-boundary value problems:

$$
\mathrm{P}_{1}\left\{\begin{array}{l}
\partial u(x, t) / \partial t=P(x, D) u(x, t), \quad t>0, \\
u(x, 0)=\phi(x), \\
B(x, D) u(x, t)=f(x, t), \quad x \in S, t>0,
\end{array}\right.
$$

and

$$
\mathbf{P}_{2}\left\{\begin{array}{l}
\partial^{2} v(x, t) / \partial t^{2}=P(x, D) v(x, t), \quad t>0, \\
v(x, 0)=0, \quad v_{t}(x, 0)=\phi(x), \\
B(x, D) v(x, t)=g(x, t), \quad x \in S, t>0 .
\end{array}\right.
$$

We assume that $B(x, D) \phi(x)$ vanishes on $S(x)=0$ and that $P(x, D) \phi(x)$ is continuous.

The interest in this paper will be in relating the solvability of $\mathrm{P}_{2}$ to $P_{1}$ and conversely by means of the Laplace transform and the inverse Laplace transform. The use of the Laplace transform will necessarily impose restrictions on the choices of the functions $f(x, t)$ and $g(x, t)$, but these conditions are satisfied in a wide class of applications. By the symbolism $\mathscr{L}_{s}^{-1}\{\psi(x, s)\}_{s \rightarrow t^{2}}$ we understand the inverse Laplace transform with the variable $s$ in the transform and the variable $t^{2}$ in the inverted function. We then have the following results:

TheOREM 1. If $\mathrm{P}_{1}$ is solvable with solution $u(x, t)$ and if

$$
g(x, t)=\Gamma(3 / 2) \mathcal{L}_{s}^{-1}\left\{s^{-8 / 2} f(x, 1 / 4 s)\right\}_{s \rightarrow t^{2}},
$$

then $\mathrm{P}_{2}$ is also solvable and

$$
v(x, t)=\Gamma(3 / 2) \mathcal{L}_{s}^{-1}\left\{s^{-8 / 2} u(x, 1 / 4 s)\right\}_{s \rightarrow t^{2}}
$$

provided the inverse Laplace transform exists in (1.1) and (1.2). 
THEOREM 2. If $\mathrm{P}_{2}$ is solvable with solution $v(x, t)$ and if

$$
f(x, t)=\frac{1}{2 \sqrt{ } \pi t^{3 / 2}} \int_{0}^{\infty} \xi e^{-\xi^{2} / 4 t} g(x, \xi) d \xi,
$$

then $\mathrm{P}_{1}$ is solvable and

$$
u(x, t)=\frac{1}{2 \sqrt{ } \pi t^{3 / 2}} \int_{0}^{\infty} \xi e^{-\xi^{2} / 4 t} v(x, \xi) d \xi
$$

provided the integrals exist in (1.3) and (1.4) for $t>0$.

The cases of $\mathrm{P}_{1}$ and $\mathrm{P}_{2}$ that are usually of interest are those in which $P(x, D)$ is an elliptic operator having a positive definite form. Then the equations in $\mathrm{P}_{1}$ and $\mathrm{P}_{2}$ are, respectively, parabolic and hyperbolic. Although the initial value problem in $\mathrm{P}_{2}$ is not well posed if $P(x, D)$ is an elliptic operator having a negative definite form, certain boundary problems related to this operator conveniently fit into our description. From the standpoint of applications, the uses of Theorems 1 and 2 are clear. A problem $\mathrm{P}_{2}$ (or $\mathrm{P}_{1}$ ) that is complicated may be transformed into a more easily solved problem $\mathrm{P}_{1}$ (or $\mathrm{P}_{2}$ ). Applications of these results will, however, be deferred to a later paper.

2. Proofs of Theorems 1 and 2. Through transformations of variables and the introduction of the Laplace transform, it will be shown that problems $P_{1}$ and $P_{2}$ can be reduced to the same problem.

Introduce the change of variables $u(x, t)=u^{*}(x, t)+\phi(x)$ and $v(x, t)=v^{*}(x, t)+t \phi(x)$, respectively, in $\mathrm{P}_{1}$ and $\mathrm{P}_{2}$. Then $\mathrm{P}_{1}$ and $\mathrm{P}_{2}$ transform, respectively, into the problems

$$
\mathrm{P}_{1}^{1}\left\{\begin{array}{l}
u_{t}^{*}(x, t)=P(x, D) u^{*}(x, t)+P(x, D) \phi(x) ; \quad u^{*}(x, 0)=0, \\
\left.\left.B(x, D) u^{*}(x, t)\right|_{s}=f(x, t) \quad \text { (since } B(x, D) \phi(x)=0 \text { on } S\right),
\end{array}\right.
$$

and

$$
\mathrm{P}_{2}^{1}\left\{\begin{array}{l}
v_{t t}^{*}(x, t)=P(x, D) v^{*}(x, t)+t P(x, D) \phi(x), \\
v^{*}(x, 0)=0, \quad v_{t}^{*}(x, 0)=0, \\
\left.B(x, D) v^{*}(x, t)\right|_{s}=g(x, t) .
\end{array}\right.
$$

In $\mathrm{P}_{2}^{1}$, introduce the change of variables $t=\tau^{1 / 2}$. Then $\mathrm{P}_{2}^{1}$ becomes

$$
\mathrm{P}_{2}^{2}\left\{\begin{array}{l}
4 \tau v_{\tau \tau}^{*}+2 v_{\tau}^{*}=P(x, D) v^{*}\left(x, \tau^{1 / 2}\right)+\tau^{1 / 2} P(x, D) \phi(x), \\
v^{*}(x, 0)=0, \quad \lim _{\tau \rightarrow 0} v_{\tau}^{*}\left(x, \tau^{1 / 2}\right)=0, \\
\left.B(x, D) v^{*}\left(x, \tau^{1 / 2}\right)\right|_{S}=g\left(x, \tau^{1 / 2}\right) .
\end{array}\right.
$$


Now introduce the Laplace transform in $\mathrm{P}_{2}^{2}$ by transforming on the variable $\tau$ with transformed variable $s$. Then $\tilde{t}^{*}(x, s)$, the Laplace transform of $v^{*}\left(x, \tau^{1 / 2}\right)$, satisfies the problem

$$
\mathrm{P}_{2}^{3}\left\{\begin{array}{l}
4 s^{2} \frac{\partial}{\partial s} \bar{v}^{*}(x, s)+6 s \bar{v}^{*}(x, s)+P(x, D) \bar{v}^{*}(x, s)+\frac{\Gamma(3 / 2)}{s^{3 / 2}} P(x, D) \phi(x)=0, \\
\left.B(x, D) \bar{v}^{*}(x, s)\right|_{s}=\bar{g}(x, s),
\end{array}\right.
$$

with $\bar{g}(x, s)$ the Laplace transform of $g\left(x, \tau^{1 / 2}\right)$. Finally, a multiplication of the equation and conditions in $\mathrm{P}_{2}^{3}$ by $s^{3 / 2} / \Gamma(3 / 2)$ leads to the problem

$$
\mathbf{P}_{2}^{4}\left\{\begin{array}{l}
4 s^{2} \frac{\partial}{\partial s}\left\{\frac{s^{3 / 2} \bar{v}^{*}}{\Gamma(3 / 2)}\right\}+P(x, D)\left\{\frac{s^{3 / 2} \bar{v}^{*}}{\Gamma(3 / 2)}\right\}+P(x, D) \phi(x)=0, \\
\left.B(x, D)\left\{\frac{s^{3 / 2}}{\Gamma(3 / 2)} \bar{v}^{*}(x, s)\right\}\right|_{s}=\frac{s^{3 / 2}}{\Gamma(3 / 2)} \bar{g}(x, s) .
\end{array}\right.
$$

In $\mathrm{P}_{1}^{1}$, introduce the change of variables $t=1 /(4 s)$ for $s>0$. Then $\mathrm{P}_{1}^{1}$ transforms into the problem

$$
\mathbf{P}_{1}^{2}\left\{\begin{array}{l}
4 s^{2} \frac{\partial}{\partial s} u^{*}(x, 1 / 4 s)+P(x, D) u^{*}(x, 1 / 4 s)+P(x, D) \phi(x)=0 \\
\left.B(x, D) u^{*}(x, 1 / 4 s)\right|_{s}=f(x, 1 / 4 s)
\end{array}\right.
$$

with $\lim _{s \rightarrow \infty} u^{*}(x, 1 / 4 s)=0$.

A comparison of $\mathrm{P}_{2}^{4}$ and $\mathrm{P}_{1}^{2}$ shows that the functions $u^{*}(x, 1 / 4 s)$ and $s^{8 / 2}\left(\bar{v}^{*}(x, s) / \Gamma(3 / 2)\right)$ satisfy (i) the same differential equation and (ii) the same boundary conditions provided that

$$
\begin{aligned}
& \text { (a) } f(x, 1 / 4 s)=\frac{s^{8 / 2}}{\Gamma(3 / 2)} \bar{g}(x, s), \\
& \text { (b) } \lim _{s \rightarrow \infty} s^{3 / 2} \bar{v}^{*}(x, s)=0 .
\end{aligned}
$$

The conditions (2.1a) are those covered by the hypotheses (1.2) and (1.4). Imposing these conditions along with (2.1b), we get

$$
i^{*}(x, s)=\Gamma(3 / 2) s^{-3 / 2} u^{*}(x, 1 / 4 s),
$$

and the result (1.1) follows by inversion and our definitions of $u^{*}$ and $v^{*}$. The result (1.3) also follows from (2.2). This completes the proof.

The requirement that $\phi(x)$ be such that $P(x, D) \phi(x)$ is continuous is not necessary. By mollifying $\phi(x)$ or interpreting $P(x, D) \phi(x)$ in the sense of distributions, the continuity requirements can be weakened. Also observe that the method of proof only depended upon the linear- 
ity and $t$ independence of the operator $P(x, D)$. This permits $P$ to be a quite general operator. Finally, if $P_{1}$ and $P_{2}$ reduce to initial value problems, a similar argument is applicable. In this case, conditions on the known function $u(x, t)$ (or $v(x, t)$ ) can be imposed on the unknown function $v(x, t)$ (or $u(x, t)$ ). With no boundary conditions on $u$ and $v$, it is no longer necessary to require that $\phi(x)$ satisfy a boundary condition.

OAKLAND UNIVERSITY

\title{
LOCALLY NICE EMBEDDINGS IN CODIMENSION THREE ${ }^{1}$
}

\author{
BY J. L. BRYANT AND C. L. SEEBECK, III
}

Communicated by O. G. Harrold, October 16, 1967

1. Introduction. Suppose that $K$ is a $k$-dimensional compactum in the interior of a topological $q$-manifold $Q, q-k \geqq 3$. Following Hempel and McMillan [3], we say that $K$ is locally nice in $Q$ if $Q-K$ is 1-ULC. Similarly, an embedding $f: K \rightarrow \operatorname{Int} Q$ is said to be locally nice if $Q-f(K)$ is 1 -ULC.

In [1] the authors showed that a locally nice embedding of a compact $k$-dimensional polyhedron $K$ into Int $Q$, where $Q$ is a PL $q$-manifold, is $\epsilon$-tame whenever $q \geqq 5$ and $2 k+2 \leqq q$. In this announcement we outline the proof that the same is true for embeddings in codimension at least three if $K$ is a compact PL manifold. Specifically, our main result is

Theorem 1. Suppose that $M$ and $Q$ are $P L$ manifolds of dimensions $m$ and $q$, respectively, with $M$ compact, $q \geqq 5$, and $q-m \geqq 3$, and $f: M \rightarrow I n t Q$ is a locally nice embedding. Then $f$ is $\epsilon$-tame.

The following two corollaries serve to demonstrate the usefulness of Theorem 1 as applied to some special locally nice embeddings.

Corollary 1.1. Suppose that $P$ is a locally tame $(q-1)$-complex in the $P L q$-manifold $Q, q \geqq 5$, and $M$ is a compact $P L$ m-manifold in Int $Q, q-m \geqq 3$, such that $M-P$ is locally tame. Then $M$ is $\epsilon$-tame.

${ }_{1}$ This research was supported in part by NSF grant GP-5458. 